\title{
Inflammation has a role in urethane-induced lung cancer in $\mathrm{C} 57 \mathrm{BL} / 6 \mathrm{~J}$ mice
}

\author{
CAI XU ${ }^{1,2^{*}}$, LINGYU ZHOU ${ }^{1,2^{*}}$, LEI LU $^{1,2}$, TING CHEN $^{3}$, SIYU WEI $^{1,2}$, XIAOJING LIN $^{1,2}$ and XUEMEI LIAN ${ }^{1,2}$ \\ ${ }^{1}$ School of Public Health and Management, Research Center for Medicine and Social Development, \\ Innovation Center for Social Risk Governance in Health; ${ }^{2}$ Center for Lipid Research, \\ Key Laboratory of Molecular Biology on Infectious Diseases Designated by the Chinese Ministry of Education, \\ Chongqing Medical University; ${ }^{3}$ Department of Prevention and Control of Chronic Diseases, \\ Center for Disease Control and Prevention in Chongqing, Chongqing 400016, P.R. China
}

Received September 4, 2015; Accepted August 1, 2016

DOI: $10.3892 / \mathrm{mmr} .2016 .5661$

\begin{abstract}
Lung cancer is a common and highly frequent cause of cancer-associated mortality worldwide. Several studies have indicated that chronic inflammation is associated with an increased risk of several types of human cancer. The lung is vulnerable to various chemical and biological insults, and persistent exposure to these factors may result in the release of several inflammatory cytokines from inflammatory cells, thus leading to chronic inflammation and a risk of lung cancer. Due to the extensive application of C57BL/6J mice in lipid metabolism-related research, it appears important to establish a lung cancer model based on C57BL/6 J mice. Therefore, the present study designed an experimental model, in which C57BL/6J mice received several injections of urethane. The study aimed to explore whether inflammation has a role in this model of lung cancer. The results demonstrated that 10 weekly intraperitoneal injections of urethane induced a $100 \%$ lung tumor incidence, and urethane-treated mice possessed higher numbers of immune cells. In addition, the expression levels of cytokines and chemokines in bronchoalveolar lavage fluid were significantly different between the two groups. Activation of the transcription factor nuclear factor- $\mathrm{\kappa B}$ was increased in the lung tissues of urethane-treated mice, and its expression was upregulated in
\end{abstract}

Correspondence to: Dr Xuemei Lian, Center for Lipid Research, Key Laboratory of Molecular Biology on Infectious Diseases Designated by the Chinese Ministry of Education, Chongqing Medical University, 1 Yixueyuan Road, Yuzhong, Chongqing 400016, P.R. China

E-mail: xuemeiliancqmu@sina.com

Abbreviations: BALF, bronchoalveolar lavage fluid; $\mathrm{AAH}$, adenomatous hyperplasia; AD, adenoma

*Contributed equally

Key words: lung cancer, multiple injection, urethane, C57BL/6J mice, inflammation a time-dependent manner. These results suggested that the accumulation of lung inflammation may be associated with the occurrence of lung cancer in C57BL/6J mice.

\section{Introduction}

Lung cancer is a common and highly frequent cause of cancer-associated mortality worldwide $(1,2)$. In addition, the occurrence of lung cancer between mice and humans is similar, particularly adenocarcinoma (3). The incidence and outcome of lung injury and inflammation are associated with the nature of the precipitating disease, and individual susceptibility (4). Genetic susceptibility has been reported to have an important role in the induction and development of lung injury in humans (5) and animals (6), and various mouse strains display a variation in susceptibility to lung tumor induction. In current lung cancer research, a urethane model of lung cancer has been widely used, particularly in BALB/c mice (7). Previous studies have demonstrated that C57BL/6J mice are not very sensitive to urethane-induced lung cancer $(8,9)$. However, with the extensive application of C57BL/6J mice in lipid metabolism-related research, it appears important to establish a lung cancer model based on C57BL/6J mice.

It has previously been reported that chronic inflammation is associated with an increased risk of several types of human cancer (10). The lung is vulnerable to various chemical and biological insults, and persistent exposure to these factors may induce the release of several inflammatory cytokines from inflammatory cells, consequently leading to chronic inflammation and an increased risk of lung cancer $(11,12)$. Inflammatory cells in the tumor microenvironment may release cytokines to directly stimulate oncogenic signaling in cancer cells, including nuclear factor $(\mathrm{NF})-\kappa \mathrm{B}$, thus promoting cancer survival and proliferation (12). The present study designed an experimental model, in which C57BL/6J mice received numerous injections of urethane. The present study aimed to determine whether inflammation has a role in this lung cancer model. The results demonstrated that accumulation of lung inflammation was associated with the occurrence of lung cancer in C57BL/6J mice. 


\section{Materials and methods}

Animals and experimental design. A total of 40 male C57BL/6J mice (age, 6-8 weeks, weight, 12.40-14.79 g; 20 per group) were obtained from the animal facility of Chongqing Medical University (Chongqing, China) and maintained in a controlled environment (12-h light/dark cycle, ad libitum access to food and water) at the laboratory animal facility of Chongqing Medical University (Chongqing, China). All methods used in the present study were approved by the Animal Care and Use Committee at Chongqing Medical University. After 3 weeks of adaptive feeding, the mice were randomly divided into two groups: The urethane group, which received intraperitoneal (i.p) injections of urethane $(1 \mathrm{mg} / \mathrm{g}$ body weight; U2500; Sigma-Aldrich; Merck Millipore, Darmstadt, Germany) freshly dissolved in $0.9 \%$ saline; or the control group, which received an equal volume of saline. The mice were treated with urethane or saline once a week for 10 consecutive weeks. At various time points, mice were sacrificed using pentobarbital sodium $(0.2 \mathrm{mg} / \mathrm{g})$, blood was collected by cardiac puncture. Simultaneously, bronchoalveolar lavage fluid (BALF) and lung tissues were carefully collected. Tumorigenesis assessments were conducted after a 15-week latency period, as described previously (13). Mice were sacrificed at various time periods: Experimental week 13 (following continuous i.p injections of urethane or saline for 10 weeks) or experimental week 28 (final point) for further studies (Fig. 1A).

Histology and immunohistochemistry. Excised mouse lungs were inflation-fixed in $4 \%$ neutral buffered paraformaldehyde overnight at $4^{\circ} \mathrm{C}$ prior to paraffin embedding. Tissues were sectioned (5 $\mu \mathrm{m}$ thick) and mounted on slides. For histological analysis, sections were counterstained with hematoxylin and eosin. For immunohistochemistry (IHC), sections were incubated with rabbit polyclonal anti-cluster of differentiation (CD)68 (cat. no. BA0461; 1:200; Wuhan Boster Biological Technology, Ltd., Wuhan, China) and rat anti-mouse lymphocyte antigen 6G (cat. no. 551459; 1:200; BD Biosciences, Franklin Lakes, NJ, United States) overnight at $4^{\circ} \mathrm{C}$. The following day, sections were washed three times in phosphate-buffered saline (PBS) and were incubated with biotinylated anti-rabbit or anti-rat immunoglobulin $\mathrm{G}$ secondary antibodies using streptavidin/peroxidase link detection kit (cat. no. SP-9001; OriGene Technologies, Inc., Beijing, China) and Polink-1 HRP detection system for rat primary antibody (cat. no. PV-6004; OriGene Technologies, Inc.); at $37^{\circ} \mathrm{C}$ for $30 \mathrm{~min}$. Tissues were then washed three times in PBS and were incubated with horseradish peroxidase (HRP)-conjugated streptavidin for $15 \mathrm{~min}$. The tissues were washed a further three times in PBS and were developed in 3,3'-diaminobenzidine (OriGene Technologies, Inc., Beijing, China) for 3-5 min until a brown color appeared. Tissues were then washed five times in water, were counterstained with hematoxylin for 15-30 sec, were washed a further five times in water, and were finally washed with serial dilutions of ethanol and xylene. Inverted microscope was used for all observations.

BALF collection and multiplex antibody array. BALF samples were collected and were analyzed, as described in a previous study (14). Briefly, BALF collection was performed using three aliquots of $1 \mathrm{ml} 0.9 \%$ saline. The BALF fraction was then separated by centrifugation at $400 \mathrm{x}$ g for $10 \mathrm{~min}$ at $4^{\circ} \mathrm{C}$. Bronchoalveolar lavage cell numbers were counted by three people blind to the experimental condition. The cell suspension was stained with Diff-Quik stain kit (Shanghai Yuanye Biotechnology Co., Ltd., Shanghai, China) in order to assess BALF inflammatory cell types in cell smears. Multiplex antibody arrays (Quantibody ${ }^{\circledR}$ Mouse Inflammation Array 1; Raybiotech, Inc., Norcross, GA, USA) were conducted on BALF supernatant fluid according to the manufacturer's protocol, and the amount of inflammatory cytokines and chemokines were determined based on a logarithmic regression standard curve.

Western blot analysis. Proteins were extracted from lung tissue homogenates using the KeyGEN Nuclear and Cytoplasmic Protein Extraction kit (Nanjing Keygen Biotech Co., Ltd., Nanjing, China). Protein concentration was quantified using a bicinchoninic acid assay kit (Dingguo Changsheng Biotechnology Co., Ltd., Beijing, China) Equal quantity $(50 \mu \mathrm{g})$ of nuclear protein were separated by sodium dodecyl sulfate-polyacrylamide gel electrophoresis (12\% resolving gel and 5\% stacking get) and were transferred to polyvinylidene fluoride membranes (EMD Millipore, Bedford, MA, USA). The membranes were then blocked for $1 \mathrm{~h}$ at $37^{\circ} \mathrm{C}$ in $5 \%$ non-fat milk, and were incubated with phosphorylated (p)-NF-кB (cat. no. 3033S; 1:1,000; Cell Signaling Technology, Inc., Danvers, MA, United States) primary antibodies overnight at $4^{\circ} \mathrm{C}$. The primary antibody was detected using a rabbit HRP-conjugated secondary antibody (cat. no. BS13278; 1:10,000; Bioworld Technology, Inc., St. Louis Park, MN, USA). The blots were visualized using a Chemiluminescent HRP Substrate (EMD Millipore). Results were quantified using Image $\mathbf{J}$ version 1.4 software (National Institutes of Health, Bethesda, MD, USA) and are presented as optical density per square millimeter. Lamin B-1 was used as control antibody (cat. no. BS3547; 1:1,000, Bioword Technology, Inc.)

Statistical analysis. All experiments were conducted three times and are reproducible. Data are presented as the mean \pm standard error of the mean. Statistical significance for each variable was estimated using Student's t-test, or two-way analysis of variance followed by least squares means multiple comparisons test using SAS version 9.2 (SAS Institute, Inc., Cary, NC, USA). $\mathrm{P}<0.05$ was considered to indicate a statistically significant difference.

\section{Results}

Effects of urethane on body weight and lung tumor incidence in C57BL/6J mice. The body weights of the experimental mice were detected once a week, up to week 28 . Body weight was reduced in the urethane-treated group compared with in the saline-treated control group during and after urethane administration (Fig. 1B). The continuous i.p injection of urethane over 10 weeks induced $100 \%$ lung tumor incidence at week 28 (no. of mice with tumors/no. of mice injected; data not shown), and tumors were particularly evident (Fig. 1C). Histopathological analysis was conducted, and the lungs of the urethane-treated group exhibited the following morphological alterations: Epithelial hyperplasia, atypical adenomatous 
A

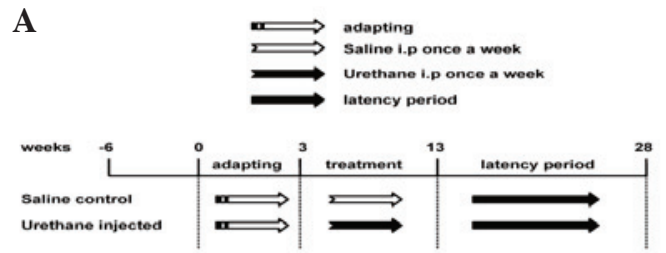

C

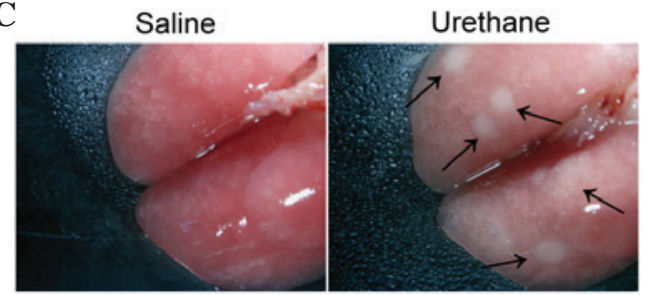

B

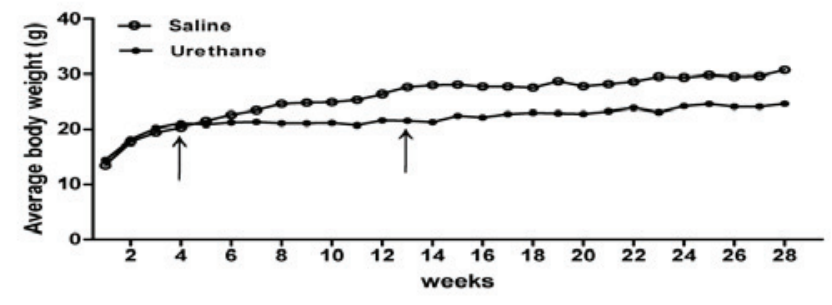

D

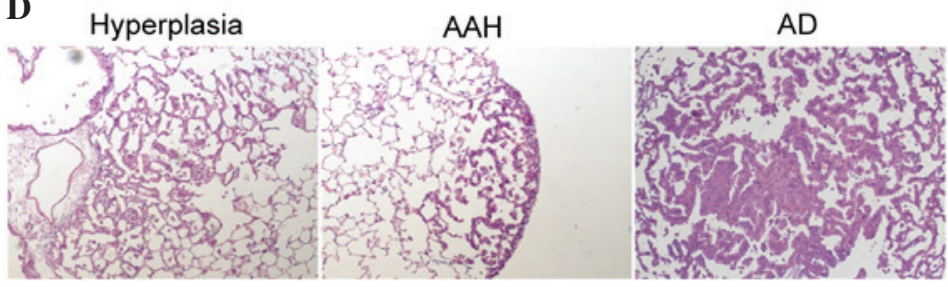

Figure 1. Differences in body weight and lung tumor incidence between the study groups. (A) Schematic diagram of the experimental design. Several urethane injections were administered to induce a lung carcinogenesis model in C57BL/6J mice. (B) Average weight of each group. Body weights were measured once a week, up to week 28. (C) Images of lungs under a dissecting microscope. Arrows indicate tumors. (D) Representative hematoxylin and eosin staining of lung sections from urethane-treated mice, exhibiting epithelial cell hyperplasia, adenomatous hyperplasia (AAH) and adenoma (AD). I.p, intraperitoneal. Magnification, x200.

A

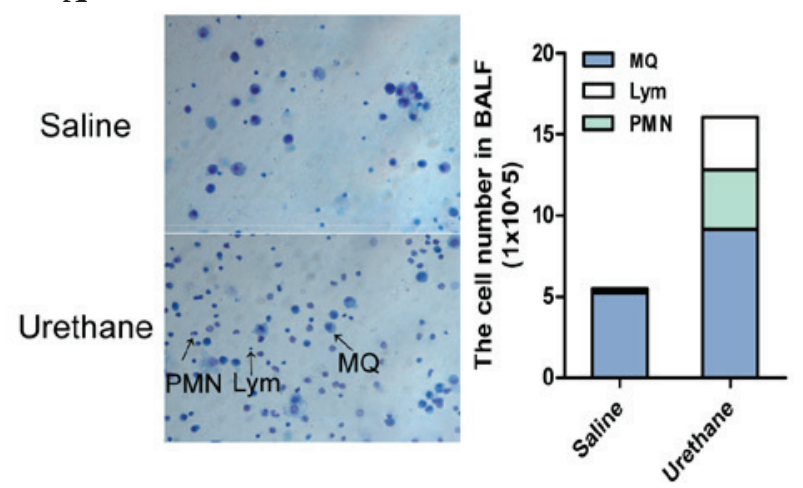

B

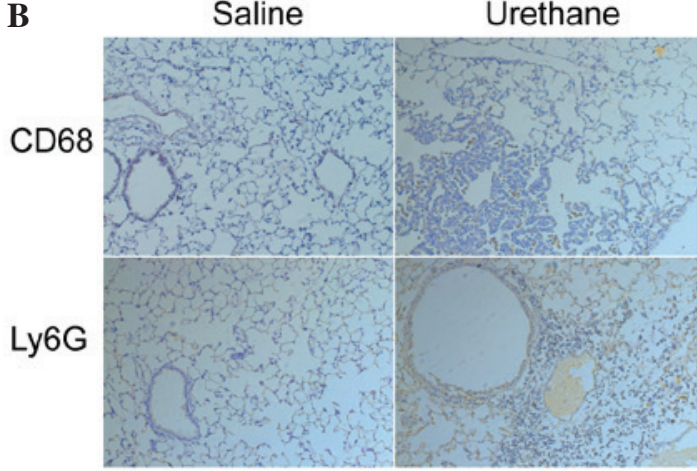

Figure 2. Alterations to the number of immune cells in bronchoalveolar lavage fluid (BALF) and lung tissue. (A) Diff-Quik staining of BALF cells (left) and the number of inflammatory cells in the BALF (right). (B) The number of macrophages and neutrophils were increased in the lung tissues of urethane-treated mice, as determined by immunohistochemistry. Magnification, x100. MQ, macrophages; Lym, lymphocytes; PMN, polymorphonuclear cells; CD68, cluster of differentiation 68; Ly6G, lymphocyte antigen 6G.

hyperplasia (AAH) and adenoma (AD) (Fig. 1D); however, no adenocarcinomas were observed at week 28.

Effects of urethane on lung inflammation. The number of immune cells in the BALF was significantly different between the urethane-treated mice and control mice at week 13. Urethane-treated mice exhibited an overall increased number of cells in the BALF (data not shown), and the number of macrophages, polymorphonuclear cells and lymphocytes were increased to varying degrees, as determined by Diff-Quik staining (Fig. 2A). Based on these results, the present study aimed to determine whether lung tissues exhibited the same changes. The results of IHC demonstrated that macrophages and neutrophils were increased in the lung tissues of urethane-treated mice compared with in saline-treated mice at week 28 (Fig. 2B).
To determine the circulating levels of cytokines and chemokines in BALF at week 13, multiplex antibody arrays were conducted. The expression levels of cytokines and chemokines in BALF were significantly different between the two groups. Several important cytokines and chemokines involved in lung inflammation were detected (Fig. 3). BALF levels of several important proinflammatory cytokines and chemokines, including interleukin (IL)-6, regulated upon activation normal T cell expressed and secreted (RANTES), thymus and activation regulated chemokine (TARC) and TIMP metallopeptidase inhibitor 1 (TIMP-1) were significantly increased in the urethane-treated group. However, the levels of anti-inflammatory cytokines and chemokines, such as IL-2, IL-4, IL-10 and IL-13 were downregulated in BALF from the urethane-treated mice compared with the controls. Furthermore, 

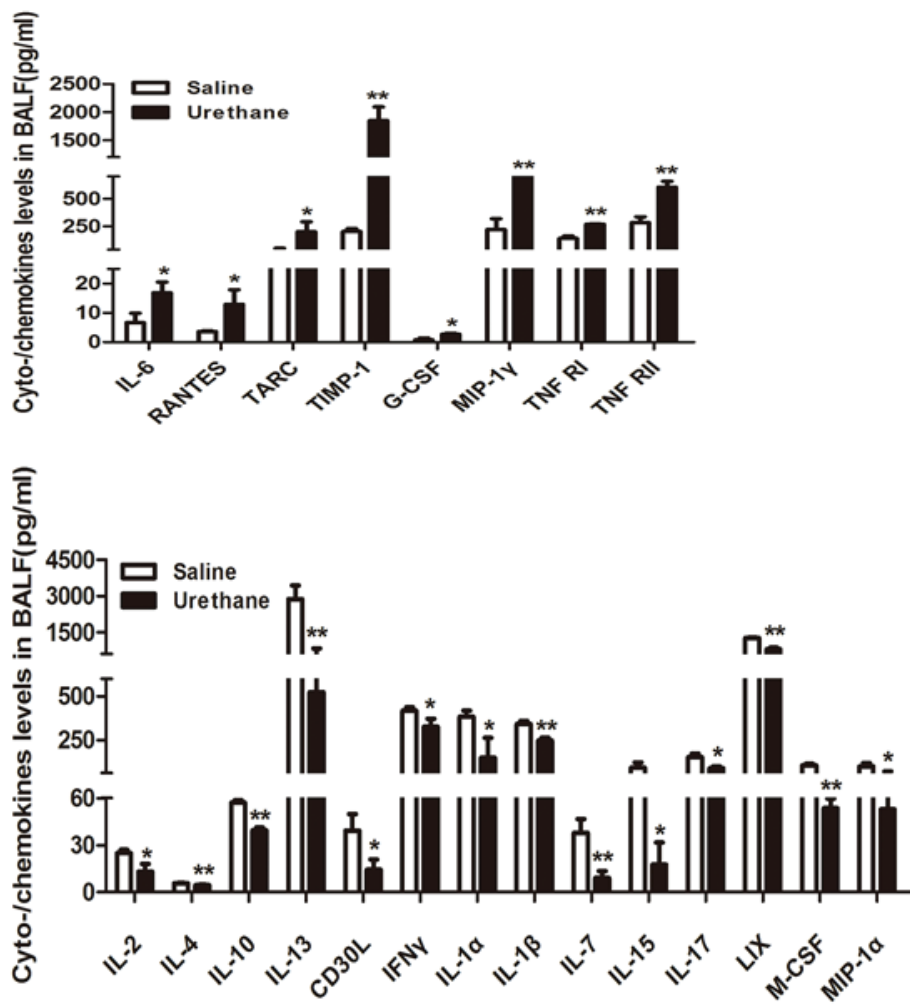

Figure 3. Multiplex antibody array quantification of several important cytokines and chemokines in bronchoalveolar lavage fluid (BALF) of the two groups ( $\mathrm{n}=3$ /group). Data are presented as the mean \pm standard error of the mean. Student's $\mathrm{t}$ test was used to determine significance. ${ }^{*} \mathrm{P}<0.05,{ }^{* * *} \mathrm{P}<0.01$ vs. saline group. IL, interleukin; RANTES, regulated upon activation normal T cell expressed and secreted; TARC, thymus and activation regulated chemokine; TIMP-1, TIMP metallopeptidase inhibitor 1; M-/G-CSF, macrophage-/granulocyte-colony stimulating factor; TNFRI/II, tumor necrosis factor receptor I/II; CD30L, cluster of differentiation 30 ligand; IFN $\gamma$, interferon $\gamma$; LIX, C-X-C motif chemokine 5; MIP, macrophage inflammatory protein.
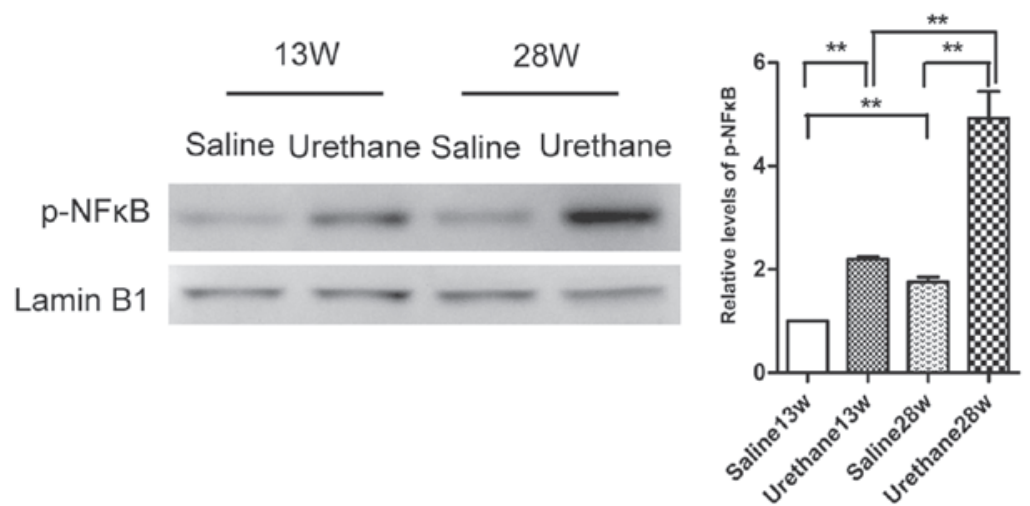

Figure 4. Western blot analysis of total phosphorylated (p)-nuclear factor (NF)-кB expression in lung tissues of urethane-treated mice. Lamin B1 was used as nuclear protein control. Densitometry quantification was analyzed among 3-5 mice per group using ImageJ software. Data are presented as the mean \pm standard error of the mean. (Saline week 13 vs. Urethane week 13: F=152.51, P<0.0001; Saline week 28 vs. Urethane week 28: F=1050.88, P<0.0001; Saline week 13 vs. Saline week 28: $\mathrm{F}=61.01, \mathrm{P}<0.0001$; Urethane week 28 vs. Urethane week 28: $\mathrm{F}=777.23, \mathrm{P}<0.0001) .{ }^{* *} \mathrm{P}<0.01$.

the levels of granulocyte-colony stimulating factor (CSF), macrophage inflammatory protein (MIP)- $1 \gamma$, tumor necrosis factor receptor (TNFR)I and TNFRII were significantly increased in urethane-treated mice, and the expression levels of CD30 ligand, interferon $\gamma$, IL-1 $\alpha$, IL-1 $\beta$, IL-7, IL-15, IL-17, $\mathrm{C}-\mathrm{X}$-C motif chemokine 5 , macrophage-CSF and MIP-1 $\alpha$ were significantly decreased compared with in the control group.
Expression of the transcription factor $N F-\kappa B$ following urethane treatment. The expression levels of $\mathrm{p}-\mathrm{NF}-\mathrm{\kappa B}$ were detected in lung tissues from the urethane-treated and saline-treated mice by western blotting at weeks 13 and 28 (Fig. 4). NF- $\mathrm{kB}$ activation was increased following urethane treatment, and the expression levels increased in a time-dependent manner. 


\section{Discussion}

Genetic background has been reported to have an important role in the induction and development of lung injury in humans (5) and animals (6), and various species of mice exhibit various levels of sensitivity to induced lung cancer. Miller et al (13) reported that a single dose of urethane does not induce a high incidence of AD in B6 mice; however, 10 weekly urethane injections induced $\sim 100 \%$ lung tumor incidence. The present study was designed to explore whether inflammation has a role in lung cancer induced by several injections of urethane in $\mathrm{C} 57 \mathrm{BL} / 6 \mathrm{~J}$ mice. The results demonstrated that the continuous i.p administration of urethane over 10 weeks induced $100 \%$ lung tumor incidence, which was consistent with a previous study (13). AAH and $\mathrm{AD}$ were the predominant morphological changes observed in the urethane model; of which AD was shown to be similar to human lung cancer.

Inflammation has a key role in cancer. Raposo et al (15) demonstrated that inflammatory cells are able to produce reactive oxygen species and reactive nitrogen intermediates, which increase the mutation rate of cells, induce DNA damage and increase genomic instability; these molecules also inactivate mismatch repair functions, thus supporting tumor promotion. Due to the close association between inflammation and cancer (16-19), the present study aimed to evaluate whether inflammatory changes appear prior to the appearance of hyperplasia or tumor. It was determined that inflammatory changes occurred at week 13; however, tumorigenesis was not observed until week 28. Redente et al (20) reported that macrophages in normal lungs were located in the alveolar interstitia and airways, whereas macrophages adjacent to tumors and neutrophil accumulation were observed in the lungs of urethane-treated A/J mice. In the present study, compared with the saline control group, urethane-treated mice exhibited increased numbers of macrophages and neutrophils (Fig. 2) in BALF and lung tissues. Among the inflammatory cell types in the lungs, macrophages are the most abundant. Depending on microenvironmental cues, these cells are able to stimulate inflammatory responses via the secretion of proinflammatory cytokines, or suppress immune responses by releasing high levels of anti-inflammatory cytokines. IL-6 is a multifunctional cytokine that may regulate immune and inflammatory responses, including control of the acute phase response at the beginning of acute inflammation, regulation of B-cell and T-cell differentiation and activation, and promotion of cell growth and survival (21). Other members of the IL family associated with lung cancer include IL-2, IL-4 and IL-10 (22). IL-2-activated NK cells are able to induce regression of established lung tumors (22). IL-4 is an anti-inflammatory cytokine, which reduces the production of proinflammatory cytokines by monocytes and with direct antiproliferative effects in some tumors (22). Additionally, it induces cathepsin protease activity in tumor-associated macrophages to promote cancer growth and invasion and stimulates 15-hydroxyprostaglandin dehydrogenase activity mediated by janus kinase-signal transducer and activator of transcription 6 , mitogen-activated protein kinase, phosphoinositide 3-kinase/Akt and protein kinase $\mathrm{C}$ signaling pathways (22). IL-10 is an immunosuppressive cytokine involved in carcinogenesis via immune escape (22). RANTES is a C-C chemokine that serves as a chemoattractant for various cells. Conti and DiGioacchino (23) identified RANTES as a mediator of inflammation. In the present study, the expression levels of proinflammatory cytokines and chemokines, including IL-6, RANTES, TARC and TIMP-1 were significantly increased in BALF from urethane-treated mice, whereas the levels of anti-inflammatory cytokines and chemokines, such as IL-2, IL-4, IL-10 and IL-13 were downregulated (Fig. 3). Chronic inflammation is a characteristic phenotype of urethane-induced tumorigenesis. Narayan and Kumar (24) reported that urethane-induced lung tumorigenesis in BALB/c mice may be caused by chronic lung inflammation.

The NF- $\kappa \mathrm{B}$ transcription factor functions as a crucial regulator of inflammation, the immune response, and cell survival $(25,26)$. In addition, $\mathrm{NF}-\kappa \mathrm{B}$ has been implicated in cellular transformation and tumorigenesis, and has been revealed to be activated at the early stages of carcinogenesis $(27,28)$. In mice treated with several injections of urethane, $N F-\kappa B$ was activated in the lung tissues, and its expression increased in a time-dependent manner (Fig. 4). These results were inconsistent with those of Stathopoulos et al (28); however, C57BL/6 mice received weekly i.p injections of urethane for only 4 weeks in this previous study. The results of the present study suggested that the duration of urethane injections may have an important role in the activation of $\mathrm{NF}-\kappa \mathrm{B}$.

In conclusion, the present study determined that the accumulation of lung inflammation was associated with the occurrence of lung cancer in C57BL/6J mice.,Due to the extensive application of $\mathrm{C} 57 \mathrm{BL} / 6 \mathrm{~J}$ mice in lipid metabolism-associated lung cancer research, multiple injections of urethane may be potential experimental animal models.

\section{Acknowledgements}

The present study was supported by grants from the Natural Science Foundation of China (NSFC, grant no. 81071907), the Natural Science Foundation of Chong Qing (CSTC, grant no. 2011BB5128) and the Program for New Century Excellent Talents in University, China (grant no. NCET-10-0997).

\section{References}

1. Jemal A, Bray F, Center MM, Ferlay J, Ward E and Forman D: Global cancer statistics. CA Cancer J Clin 61: 69-90, 2011.

2. Siegel R, Ma J, Zou Z and Jemal A: Cancer statistics, 2014. CA Cancer J Clin 64: 9-29, 2014.

3. Malkinson AM: Molecular comparison of human and mouse pulmonary adenocarcinomas. Exp Lung Res 24: 541-555, 1998.

4. Nonas S, Miller I, Kawkitinarong K, Chatchavalvanich S, Gorshkova I, Bochkov VN, Leitinger N, Natarajan V, Garcia JG and Birukov KG: Oxidized phospholipids reduce vascular leak and inflammation in rat model of acute lung injury. Am J Respir Crit Care Med 173: 1130-1138, 2006.

5. Lagan AL, Melley DD, Evans TW and Quinlan GJ: Pathogenesis of the systemic inflammatory syndrome and acute lung injury: Role of iron mobilization and decompartmentalization. Am J Physiol Lung Cell Mol Physiol 294: L161-L174, 2008.

6. Moraes TJ, Zurawska JH and Downey GP: Neutrophil granule contents in the pathogenesis of lung injury. Curr Opin Hematol 13: 21-27, 2006

7. Malkinson AM and Beer DS: Major effect on susceptibility to urethan-induced pulmonary adenoma by a single gene in BALB/cBy mice. J Natl Cancer Inst 70: 931-936, 1983. 
8. Bernert H, Sekikawa K, Radcliffe RA, Iraqi F, You M and Malkinson AM: Tnfa and Il-10 deficiencies have contrasting effects on lung tumor susceptibility: Gender-dependent modulation of IL-10 haploinsufficiency. Mol Carcinog 38: 117-123, 2003.

9. Doris K, Karabela SP, Kairi CA, Simoes DC, Roussos C, Zakynthinos SG, Kalomenidis I, Blackwell TS and Stathopoulos GT: Allergic inflammation does not impact chemical-induced carcinogenesis in the lungs of mice. Respir Res 11: 118, 2010.

10. Bartsch $\mathrm{H}$ and Nair J: Chronic inflammation and oxidative stress in the genesis and perpetuation of cancer: Role of lipid peroxidation, DNA damage, and repair. Langenbecks Arch Surg 391: 499-510, 2006.

11. Azad N, Rojanasakul Y and Vallyathan V: Inflammation and lung cancer: Roles of reactive oxygen/nitrogen species. J Toxicol Environ Health B Crit Rev 11: 1-15, 2008.

12. Candido $\mathrm{J}$ and Hagemann T: Cancer-related inflammation. J Clin Immunol 33 (Suppl 1): S79-S84, 2013.

13. Miller YE, Dwyer-Nield LD, Keith RL, Le M, Franklin WA and Malkinson AM: Induction of a high incidence of lung tumors in C57BL/6 mice with multiple ethyl carbamate injections. Cancer Lett 198: 139-144, 2003.

14. Lian X, Qin Y, Hossain SA, Yang L, White A, Xu H, Shipley JM, Li T, Senior RM, Du H and Yan C: Overexpression of Stat $3 \mathrm{C}$ in pulmonary epithelium protects against hyperoxic lung injury. J Immunol 174: 7250-7256, 2005.

15. Raposo TP, Beirão BC, Pang LY, Queiroga FL and Argyle DJ: Inflammation and cancer: Till death tears them apart. Vet J 205: $161-174,2015$

16. Kanterman J, Sade-Feldman M and Baniyash M: New insights into chronic inflammation-induced immunosuppression. Semin Cancer Biol 22: 307-318, 2012.

17. Gonda TA, Tu S and Wang TC: Chronic inflammation, the tumor microenvironment and carcinogenesis. Cell Cycle 8: 2005-2013, 2009.
18. Albini A and Sporn MB: The tumour microenvironment as a target for chemoprevention. Nat Rev Cancer 7: 139-147, 2007.

19. Porta C, Larghi P, Rimoldi M, Totaro MG, Allavena P, Mantovani A and Sica A: Cellular and molecular pathways linking inflammation and cancer. Immunobiology 214: 761-777, 2009.

20. Redente EF, Orlicky DJ, Bouchard RJ and Malkinson AM: Tumor signaling to the bone marrow changes the phenotype of monocytes and pulmonary macrophages during urethane-induced primary lung tumorigenesis in A/J mice. Am J Pathol 170: 693-708, 2007.

21. Nguyen DP, Li J and Tewari AK: Inflammation and prostate cancer: The role of interleukin 6 (IL-6). BJU Int 113: 986-992, 2014.

22. Cho WC, Kwan CK, Yau S, So PP, Poon PC and Au JS: The role of inflammation in the pathogenesis of lung cancer. Expert Opin Ther Targets 15: 1127-1137, 2011.

23. Conti P and DiGioacchino M: MCP-1 and RANTES are mediators of acute and chronic inflammation. Allergy Asthma Proc 22: 133-137, 2001.

24. Narayan C and Kumar A: Constitutive over expression of IL-1 $\beta$, IL-6, NF- $\mathrm{KB}$, and Stat 3 is a potential cause of lung tumorgenesis in urethane (ethyl carbamate) induced Balb/c mice. J Carcinog 11: 9,2012.

25. Hayden MS and Ghosh S: Signaling to NF-kappaB. Genes Dev 18: 2195-2224, 2004.

26. Baker RG, Hayden MS and Ghosh S: NF- $\kappa B$, inflammation, and metabolic disease. Cell Metab 13: 11-22, 2011.

27. Pikarsky E, Porat RM, Stein I, Abramovitch R, Amit S, Kasem S, Gutkovich-Pyest E, Urieli-Shoval S, Galun E and Ben-Neriah Y: NF-kappaB functions as a tumour promoter in inflammation-associated cancer. Nature 431: 461-466, 2004.

28. Stathopoulos GT, Sherrill TP, Cheng DS, Scoggins RM, Han W, Polosukhin VV, Connelly L, Yull FE, Fingleton B and Blackwell TS: Epithelial NF-kappaB activation promotes urethane-induced lung carcinogenesis. Proc Natl Acad Sci USA 104: 18514-18519, 2007. 